\title{
STROKE - CORRELATIONS BETWEEN CLINICAL EXAMINATION, IMAGING FINDINGS AND MICROSCOPICAL ASPECTS
}

\author{
Anca Hancu', Mariana Deacu², Madalina Bosoteanu' \\ ${ }^{1}$ Neurology Department, Constanta County Emergency Hospital \\ ${ }^{2}$ Pathology Department, Constanta County Emergency Hospital
}

\begin{abstract}
Stroke is the most frequent cause of disability among the neurologic diseases of the adult. In the last two decades, the extraordinary development of brain imaging techniques allows the rapid recognition of normal, ischemic, infarction or hemorrhagic brain tissue.

The term cerebrovascular disease designates any abnormality of the brain resulting from a pathological process of the blood vessels, including occlusion by embolus or thrombus, rupture of a vessel, an altered permeability of the vessel wall, or increased viscosity or other changes in quality of the blood flowing through the cerebral vessels. The clinical, imagistic and histological correlations in the study of arteriosclerosis are very important due to the fact that cardiovascular disease and stroke are the main causes of death in developped countries.

Our study consists of a group of 36 patients admitted in the Neurology department of Constanta County Emergency Hospital in the first six months of 2012 with the diagnosis of acute stroke, which died within the first 5 days from admission. The studied group was divided into four subgroups, according to the CT scan findings and clinical examination on admission and the autopsy results.

In the sudden death cases that had no acute lesions on the brain CT on admission, histological examination revealed the diagnosis. In all cases, histological examination included in showed atherosclerotic modifications of cerebral and peripheral vessels (the circle of Willis arteries, coronary arteries, aorta and renal arteries).
\end{abstract}

Keywords: atherosclerosis, CT scan, histological examination, stroke

\section{INTRODUCTION}

Stroke is the most frequent cause of disability as frequency and importance among the neurologic diseases of the adult. In the last two decades, the extraordinary development of brain imaging techniques allows the rapid recognition of normal, ischemic, infarction or hemorrhagic brain tissue (1).

The term cerebrovascular disease (stroke) designates any abnormality of the brain resulting from a pathologic process of the blood vessels, including occlusion of the lumen by embolus or thrombus, rupture of a vessel, an altered permeability of the vessel wall, or increased viscosity or other change in quality of the blood flowing through the cerebral vessels.

The vascular pathologic process may be considered not only in its grosser aspects - embolism, thrombosis, dissection, or rupture of a vessel - but also in terms of more basic or primary disorder, i.e., atherosclerosis, hypertensive arteriosclerotic change, arteritis, aneurismal dilatation, and developmental malformation.

These are two main types - ischemia, with or without infarction, and hemorrhage - and unless one or the other occurs, the vascular lesion usually remains silent.

Arteriosclerosis means thickening and loss of elasticity of the arterial walls.

There are 3 patterns of arteriosclerosis:

1. atherosclerosis: affects the large and medium sized arteries with formation of atheromatous plaques

2. Monckeberg medial calcific sclerosis: at the level of muscular arteries 
3. arteriolosclerosis: at the level of small arteries and arterioles; is a common aging phenomenon; in the brain the so-called small vessel ischaemic disease can cause lacunar infarcts and hemmorhages.

Atherosclerosis (the name is derived from the greek sclerosis - endured and athere - mash) means the thickening of the arterial intima by lipid accumulation. The atherosclerosis of the central nervous system vessels causes transient cerebral ischemia and strokes. It develops focally in time and space. The atheroma is usually defined as an stenosing lesion. Atherosclerosis can cause ectasies and aneurismal disease (2).

The clinical, imagistic and histological correlations in the study of arteriosclerosis are very important due to the fact that cardiovascular disease and stroke are the main causes of death in developped countries.

Imaging evaluations performed by $\mathrm{CT}$ scan demonstrates and accurately localizes even small hemorrhages, hemorrhagic infarcts, subarachnoid blood, aneurysms, arterio-venous malformations and established regions of infarction. $(3,4)$

Clinical examination revealed: rapid onset, neurologic deficits and, more rarely, the presence of excitatory manifestations. Stroke in carotid system presents: hemiparesis, hemiplegia, sensory deficits, aphasia, monocular blindness, homonymous lateral right or left hemianopsia, epileptic seizures. Stroke in vertebro-basilary system presents: vertigo, pyramidal syndrome, sensory deficit, bilateral visual impairment, cranial nerves paralysis (alternating hemiplegia), ocular motility impairment, cerebellar syndrome, involuntary movements $(5,15)$.

\section{MATERIAL AND METHOD}

We studied a group of 36 patients from the Neurology Department of the Emergency County Hospital of Constanta deceased because of a stroke in the first 5 days from admission, in the first six months of 2012. At the end, some of the patients had another diagnosis (myocardial infarctions or pulmonary embolus), so better to say deceased because of a probable stroke.

Our study group comprises both male and female patients between the ages of 50 and 89 , as shown in Graphic 1.

Our study design was longitudinal, indirect retrospective and direct prospective.

The methodology was based on the actualization of the information regarding each clinical entity and of the medical act.

The sources of information for the studied cases were the clinical neurological charts, that contained the clinical exams, brain CT scan results and the autopsy results.

The imaging study was represented only by the brain CT scan performed on admission.

The studied group was divided into four subgroups, according with CT scan findings and clinical exam on admission:

- The first group consists of 8 patients with brain infarction;

- The second group consists of 11 patients with intraparenchymal hemorrhages, with or without ventricular extension;

- The third group consists of 6 patients with clinical diagnosis of acute stroke, stroke sequelae and brain atrophy;

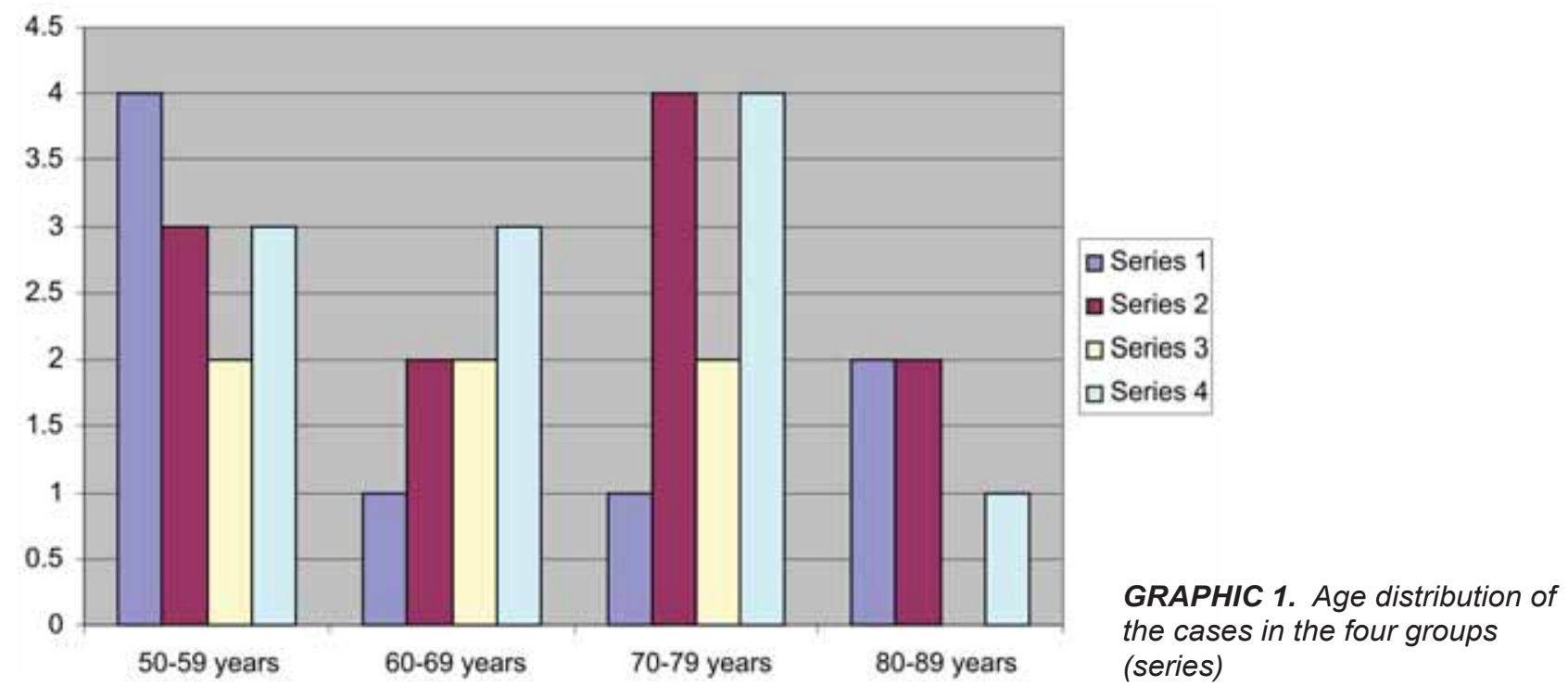


- The fourth group consists of 11 patients with a severe acute state with suspicion of stroke, with brain atrophy.

\section{RESULTS}

The first group consisted of 8 patients with acute brain infarction revealed by brain CT scan on admission.

Clinically, these patients presented left or right hemiplegia, altered consciousness and half of them were in a coma.

CT scan performed in the first 24 hours revealed:

- Hypodensity areas in the territories of the deep right middle cerebral artery -5 cases, left middle cerebral artery -2 cases and right posterior cerebral artery -1 case;

- Ventriculomegaly - 2 cases;

- Mixed (cortical - sub cortical) brain atrophy-2 cases;

- An area of old hypodensity in left frontotemporal region, both cortical and sub cortical - 1 case.

The histological examination of these cases showed wide cerebral infarction in the middle cerebral artery territory (in one case, which died on the 4th day from admission, the infarction extended to the pons and cerebellum). All the cases in the first group presented Willis circle arteries atheromatosis, calcifications of the aortic arch and, in two cases, ulcerated aortic plaques.

Heart abnormalities found in this group were: concentric hypertrophy associated with myocardial dystrophy, left ventricular hypertrophy and old myocardial infarction.

In one case with cortical - sub cortical infarction of the posterior cerebral artery teritory and with cortical brain atrophy, the histological examination also found an acute myocardial infarction, pulmonary thromboembolism, nephroangiosclerosis and liver dystrophy (this 57 year old male, therapeutically neglected hypertensive, died in the $5^{\text {th }}$ day from clinical onset).

The second group consisted of 11 cases of intraparenchimal hemorrhages, with or without ventricular extension.

Clinically, half of them presented increased cranial pressure signs with fast progression to coma, and the others presented with comatose state with rapid progression.

Brain CT scan showed the localization and the volume of intraparenchymal hematomas, the presence of mass effect, associated subarachnoid hemorrhage, calcified blood vessels, presence of brain edema and hydrocephaly. This investigation permitted to differentiate ischemic from hemorrhagic stroke and traumatic hematomas from non-traumatic ones. Small hematomas surrounded by large areas of edema rise the question of a brain tumor.

Some of the cases had hemorrhagic extension of the entire ventricular system. The patients survived for 1 to 3 days from clinical onset.

Histological examination showed atheromatosis of the arteries of Willis circle, aortic atheromatosis, edema and meningeal hemorrhages, hepatic cirrhosis in 2 cases, myocardial infarction in just one case (associated with pulmonary infarct) and one case with old myocardial infarction. The two cases with cerebral hemorrhage with a volume less than $20 \mathrm{ml}$ had acute myocardial infarction (male 69 years old with arterial hypertension under treatment for active pulmonary TB and 54 years old female with hepatic cirrhosis, arterial hypertension and chronic alcoholism).

The third group consisted of 6 cases, admitted with the clinical diagnosis of acute stroke. This group should be divided in the first two subgroups: ischemic and hemorrhagic stroke

Histological examination showed the followings:

- Bilateral ischemic lesions in the middle cerebral artery territory -3 cases;

- Large middle cerebral artery hemorrhagic infarction - 1 case;

- Brain stem hemorrhage -2 cases.

Two of these cases also presented an acute coronary infarction.

The fourth group consisted of 11 cases admitted in a severe clinical state, with the suspicion of stroke.

Brain CT scan only revealed the presence of brain atrophy in some of the cases.

Brain CT scan reveals followings:

- Mixed brain atrophy (cortico-subcortical) in 5 cases;

- Cortical atrophy (dilated brain sulci due to atrophy with normal ventricles) 1 case;

- Sub-cortical brain atrophy (communicating hydrocephalus) 1 case;

- Mixed brain atrophy associated with leukoaraiosis: 2 cases;

- Normal aspect of CT scan in 2 cases.

Clinically, one of the cases had the features of Wallenberg syndrome which was demonstrated later on histological examination and the second one had a brain stem hemorrhage with systemic atherosclerosis, nephroangiosclerosis and cardiac and central respiratory insufficiency. 
Histological examinations revealed the following in the same patients:

- In the first group: acute large middle cerebral artery ischemic lesions (5 cases), pulmonary thromboembolism and acute middle cerebral artery infarction ( 1 case) and brain stem ischemic stroke ( 1 case).

- In the second group: brain stem hemorrhagic stroke: 1 case.

- In the third groupe: acute myocardial infarction ( 2 cases) and pulmonary thromboembolism (1 case).

\section{DISCUSSION}

In the first group, the correspondence between clinical presentation, CT scan and pathology exams was tight (6).

Histological examination showed the atherosclerotic lesions spreading in the circle of Willis arteries (but also in the aorta, coronary and renal arteries) and peripheral vascular disease. However, in three of the cases (aged 50 to 59) severe atheromatosis of the aorta, myocardium or peripheral vessels were not been founded; similar findings were previously reported by other authors (6).

In this first group, the brain infarction revealed by the histological examination was significantly wider than CT image, extending to territories that the CT scan usually do not properly explore (pons and cerebellum in a case of middle cerebral artery infarction) (6).

\section{Clinical case no. 1}

We present the case of an 82 year old male, hypertensive, which was admitted to the Neurology department with a hypertensive crisis and sudden onset of left hemiplegia.

Emergency brain CT scan (Fig. 1) showed right middle cerebral artery infarction, mixed brain atrophy and leukoaraiosis. Death occurred on the $5^{\text {th }}$ day of admission.

Histological diagnosis was: right parietal lobe ischemic infarct (Fig. 2)

In the second group, brain CT scan was a very important tool in predicting the prognosis of cerebral hemorrhage. As known, any hematoma located in the putamen, thalamus or cerebellum, with a diameter of more than $3 \mathrm{~cm}$, has an increased risk of death $(6,8)$. This risk is also associated with large volume lobar hematomas (more than $50 \mathrm{~mm}$ ), followed by medium size hematomas (between 20 and $50 \mathrm{~mm}$ ) (9). Evolution of the patients with cerebellar hematomas associated with edema and com-

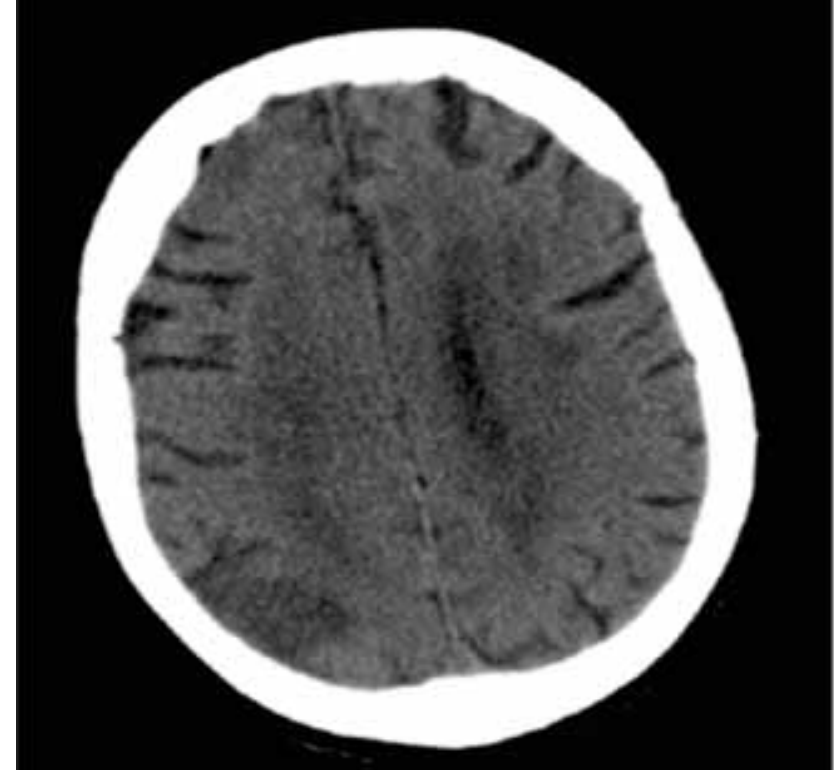

FIGURE 1. Area of moderate hyperdensity in the right parietal region, mixed brain atrophy, leukoaraiosis

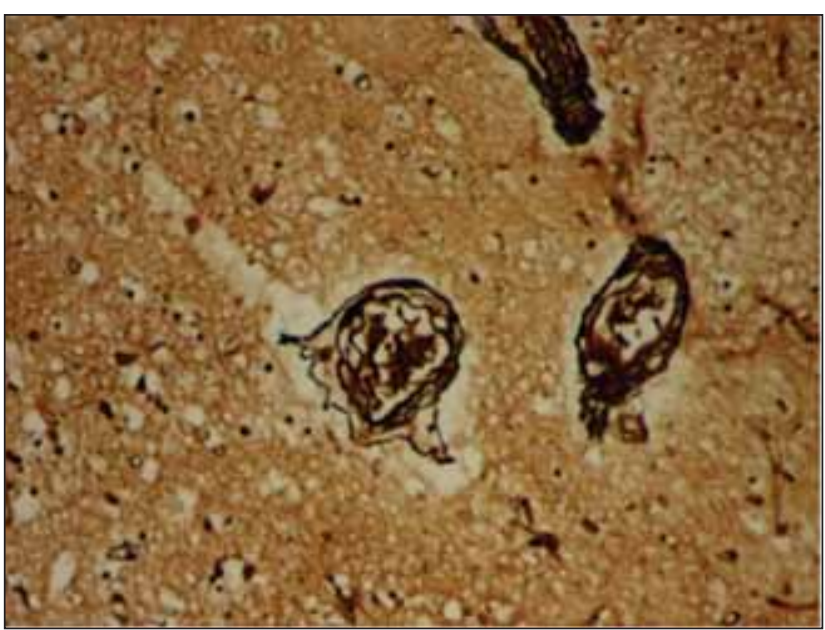

FIGURE 2. Diffuse perineuronal edema with arteriolar and capillary hyperemia and subendotheliitis, with an increased number of reticulin fibers of tunica media of the vessels (silver stain $\times 100$ )

pression of the fourth ventricle was unfavorable. The presence of hydrocephaly and intraventricular hemorrhage are also predictors for an unfavorable prognosis; similar findings were previously reported by other authors (10).

\section{Clinical case no. 2}

A case of a 76 year old male, admitted with arterial hypertension, right middle cerebral artery hemorrhage, rapid onset with loss of consciousness, head trauma, coma and rapid worsening.

CT scan (Fig. 3) shows: acute right intracerebral hemorrhage with a diameter of 5/6/6 cm $(90 \mathrm{ml})$ with mass effect on the right lateral ventricle, mixed brain atrophy, left parietal occipital post-traumatic epicranial hematoma. 


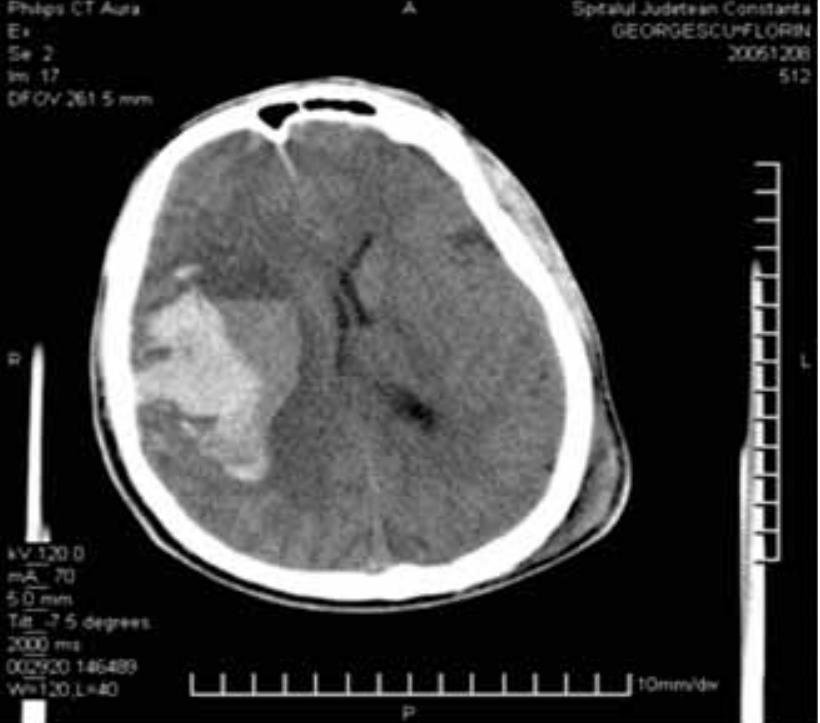

FIGURE 3. Acute right intracerebral hemorrhage

The patient died in the 5th day from admission.

Histological examination (Fig. 4 and 5): edema and diffuse meningo-cerebral hyperemia, cerebral atherosclerosis and massive cerebral hemorrhage at the level of right cerebral hemisphere with diffuse extension upon right occipital lobe and cerebellum.

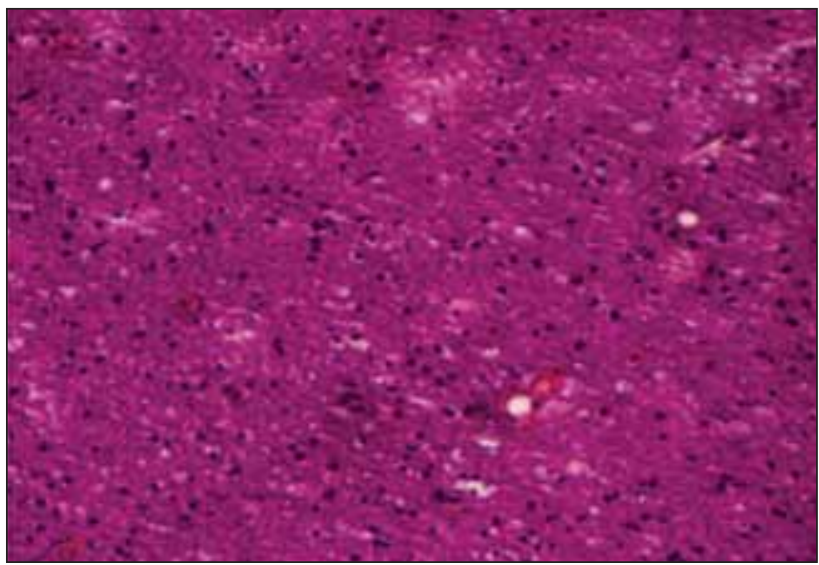

FIGURE 4. Coagulation necrosis surrounds hemorrhagic zone and consist in tisular eosinophilia with disappearance of cellular details. (H.E. X 100)

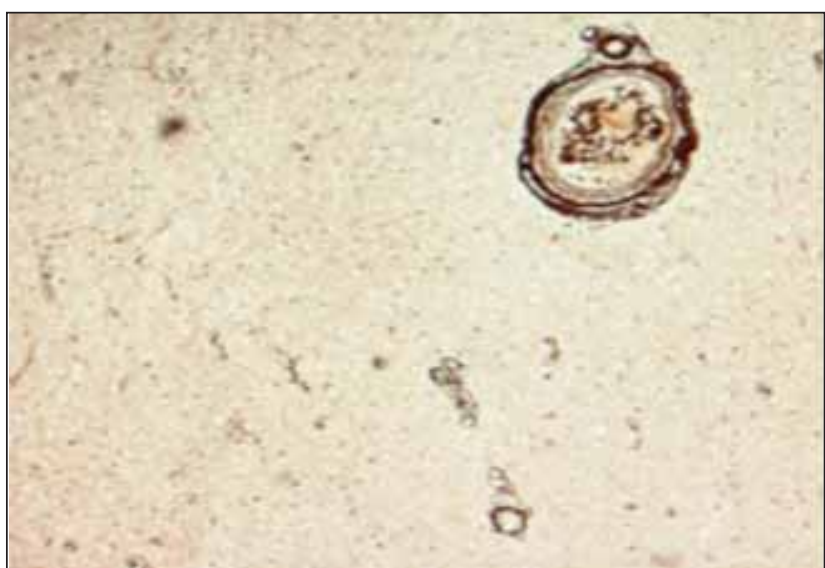

FIGURE 5. Perineural and perivascular edema with vacuolar aspect of brain substance; reticuline fibers in the vascular walls (silver stain $\times 100$ )

\section{Clinical case no. 3}

A case of a 78 year old male, admitted for vascular coma, GCS $=3$.

Emergency brain CT scan (Fig.6 a, b): acute hemorrhagic mass with $4 / 3 / 3 \mathrm{~cm}$ diameter $(30 \mathrm{ml}$ volume), localized in the deep right middle cerebral artery area, with extension of all the ventricular system, with midline deviation. He died 24 hours from admission.
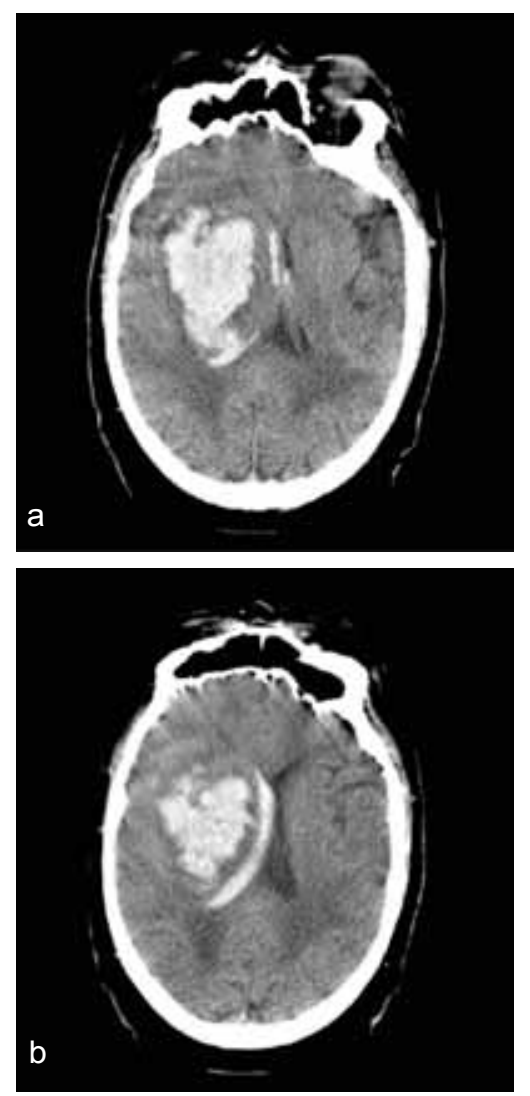

FIGURE 6 ( $a, b)$.

Acute hemorrhagic mass with $4 / 3 / 3 \mathrm{~cm}$ diameter $(30 \mathrm{ml}$ volume), localized in the deep right middle cerebral artery area

Histological examination reveals the following diagnosis: massive cerebral hemorrhage with intraventricular bleeding and cerebral atherosclerosis (Fig. 7, 8, 9).

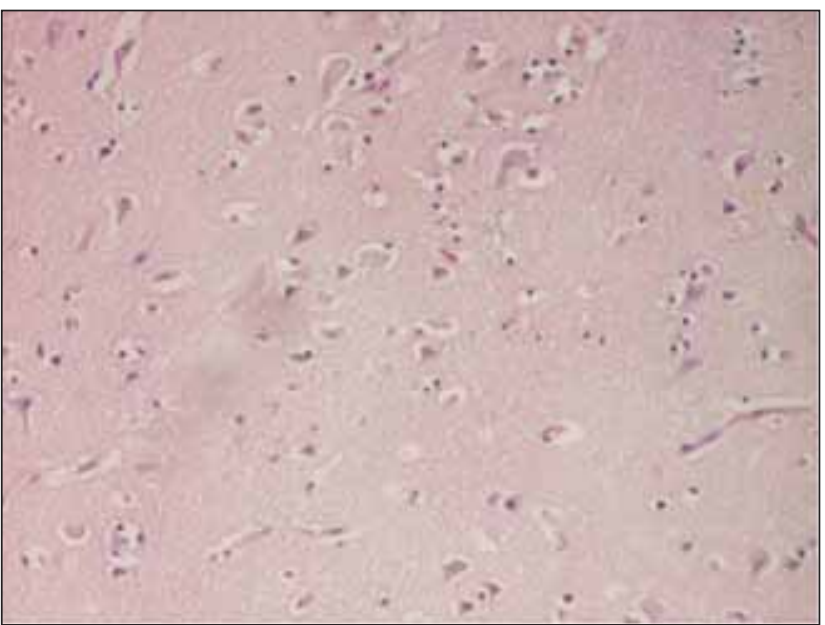

FIGURE 7. Cellular vacuolar aspect and presence of granular bodies; moderate degree of edema (H.E. X 40) 


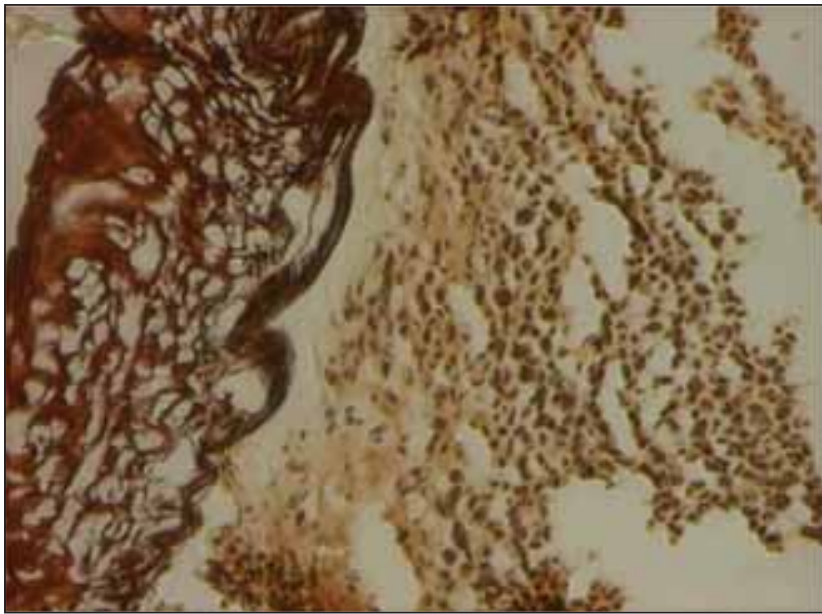

FIGURE 8. Numerous reticulin fibers in the arterial wall (Van Gieson X 200)

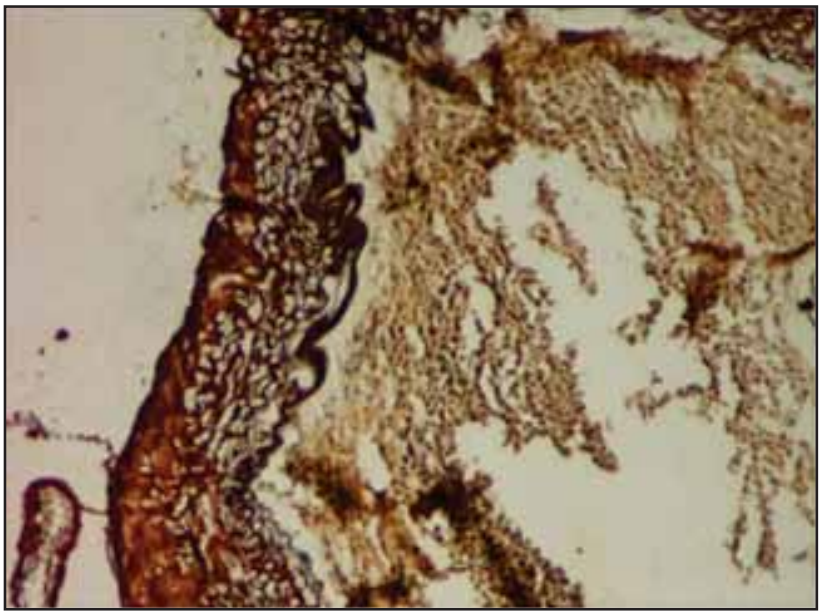

FIGURE 9. Endothelial hyperplasia with partial lumen obstruction (Van Gieson X 100)

Regarding the subjects of the third group, the diagnosis of acute stroke was evoked by the clinical course of the disease, despite the lack of typical CT scan signs. This can be explained by the known limitations of CT scan performed in the first 48 hours from the clinical onset of acute stroke in decealing the cerebral infarction. In the case of brain stem infarction, CT scan is not very useful due to movement artifacts, bony structure of the posterior fossa and small dimension of most of the infarcts in this region. $(1,3,4)$

In histological examination, these acute lesions were associated with cerebro-vascular sequelae and mixed brain atrophy, demonstrated also by CT scan, together with aortic atheromatosis with ulceration and calcifications, atheromatosis of arteries in the circle of Willis, pulmonary micro-thromboembolisms and pulmonary edema, etc. (11)

The histological examination in the cases with hemiplegia showed acute lesions in the middle cerebral arteries territories and wide middle cerebral artery hemorrhagic infarct.
The two cases of coma had a pathology correspondent: a brain stem hemorrhage in one case and a myocardial infarction in the second case $(5,12)$.

\section{Clinical case no. 4}

A case of a 66 year old male, admitted for generalized epileptic seizures followed by comatose state, with rapid progression.

Emergency brain CT scan (Fig. 10): hypodense mass in the left paramedian occipital region, old left posterior cerebral artery infarct, important brain atrophy.

The patient died in the $5^{\text {th }}$ day from admission.

Histological examinations (Fig. 11): brain edema, brain atrophy with old left occipital infarct zone but also at the level of brain stem with cystic aspect of $11 \mathrm{~cm}$.

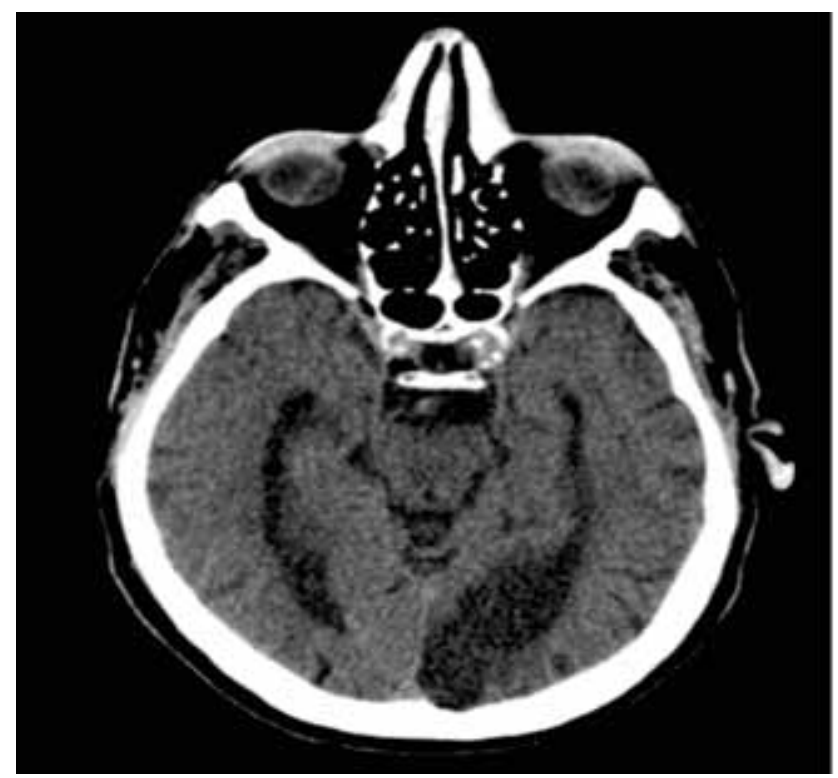

FIGURE 10. In the left paramedian occipital region, old left posterior cerebral artery infarct, important brain atrophy

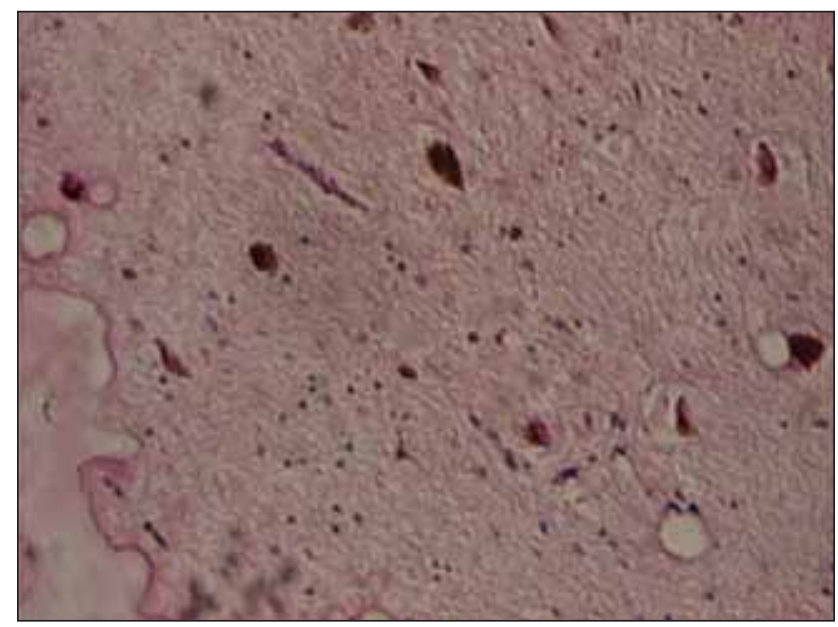

FIGURE 11. Microscopic aspect of cystic lesion points the presence of hemosiderin laden macrophages (H.E. X 100) 


\section{Clinical case no. 5}

A case of a 59 year old female, admitted for mild coma, arterial hypertension and acute right hemiplegia.

Emergency brain CT scan (Fig. 12): left intraaxial occipital hypodensity, probably an old infarction with mixed brain atrophy. Survived just 24 hours.

Histological examinations:

- Atheromatosis of the Willis circle arteries, brain edema and atrophy and white matter petechial hemorrhages (Fig. 13).

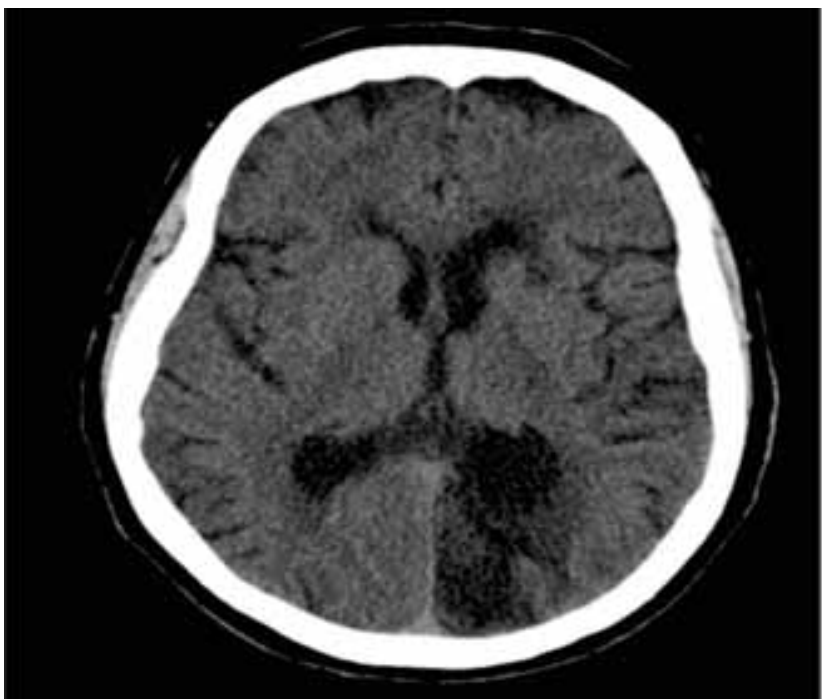

FIGURE 12. Left intra-axial occipital hypodensity, probably an old infarction with mixed brain atrophy

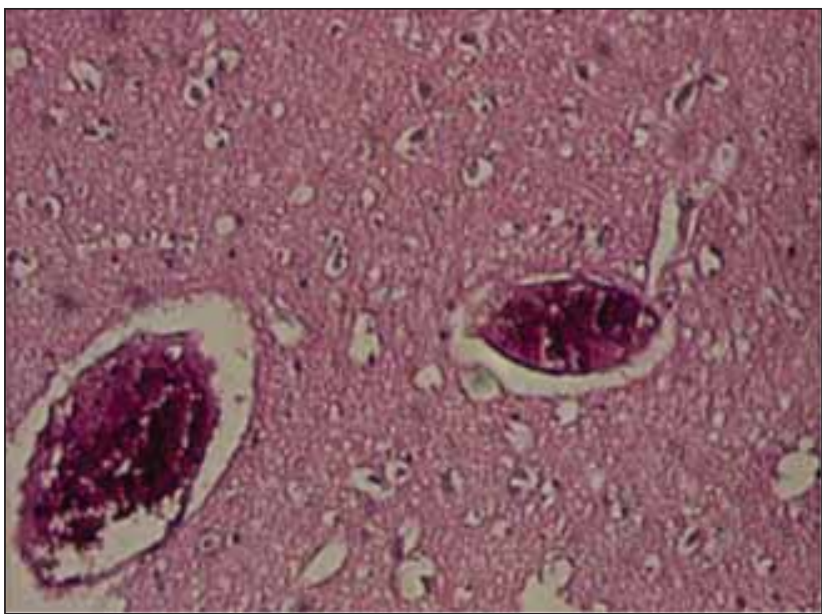

FIGURE 13. Marked capillary hyperemia with gross petechial appeareance (H.E. X 100)

In the 9 cases of the fourth group (admitted in a severe state, with important brain atrophy and leukoaraiosis showed on CT scan), the suspicion of stroke was confirmed by the histological examination in only 6 of the cases; in the other 3 cases, the final diagnosis was myocardial infarctions or pulmonary embolus. In the 2 cases with normal CT scans, the histological examination elucidated the diagnosis: brain stem ischemic stroke and brain stem hemorrhage.

As reported before, in all cases, presence of leukoaraiosis was associated with increased risk of stroke recurrence $(13,14)$.

\section{Clinical case no. 6}

The case of a 55 year old male, admitted for sudden onset coma, tetraplegia, no acute lesions on brain CT scan (Fig. 14). Death occurs on the $3^{\text {rd }}$ day from admission and the histological examination showed acute brain stem stroke. (Fig. 15).

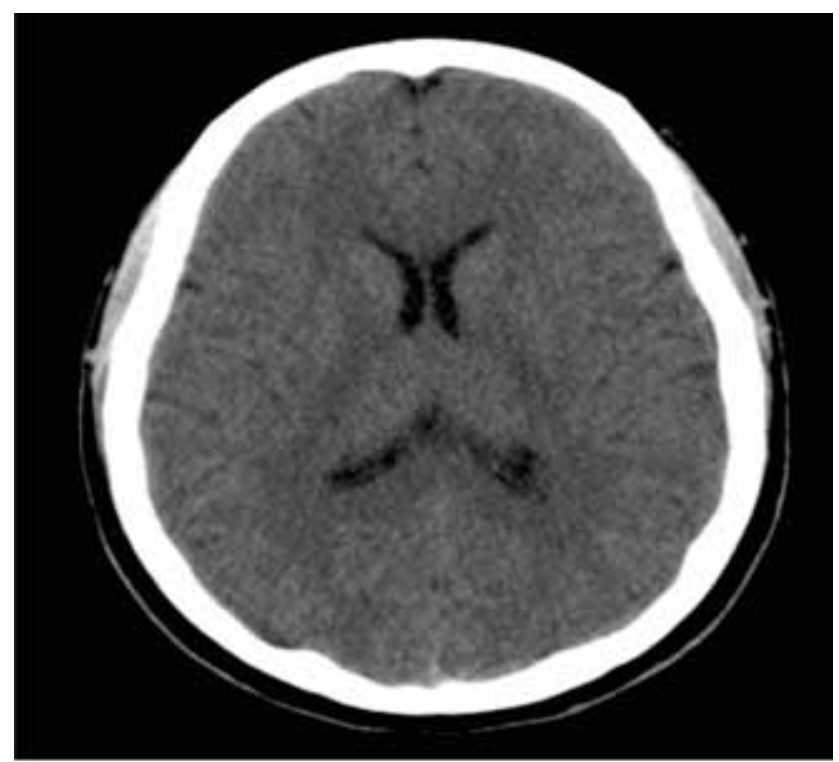

FIGURE 14. CT scan with no acute lesions

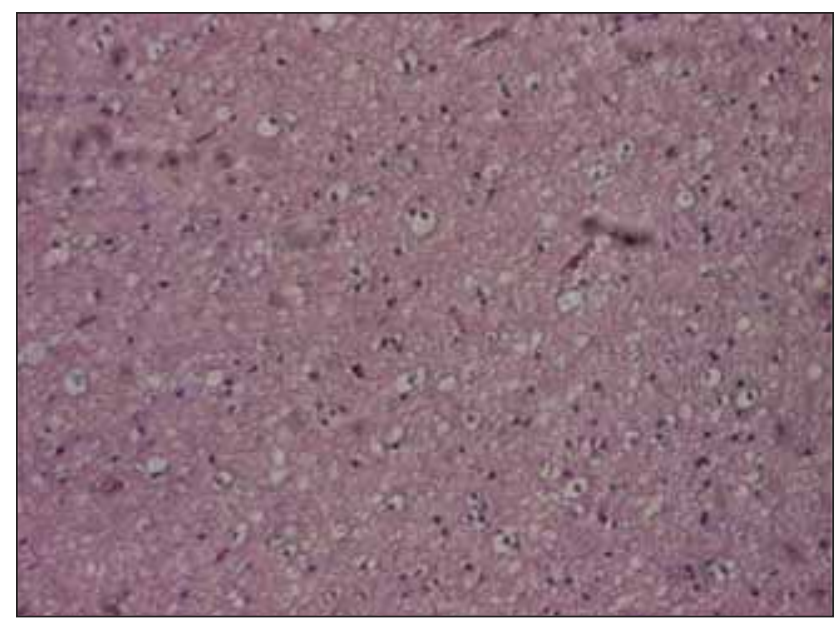

FIGURE 15. Normal appearance of neuronal nuclei (H.E. X 100)

\section{Clinical case no. 7}

The case of a 69 year old patient with mixed brain atrophy (Fig. 16), admitted for a meningeal syndrome, shortly followed by deep comatose state and death in the first 24 hours. 
Histological examination (Fig. 17, 18):

- Atheromatosis of Willis circle arteries, meningo-cerebral edema and internal hydrocephalus.

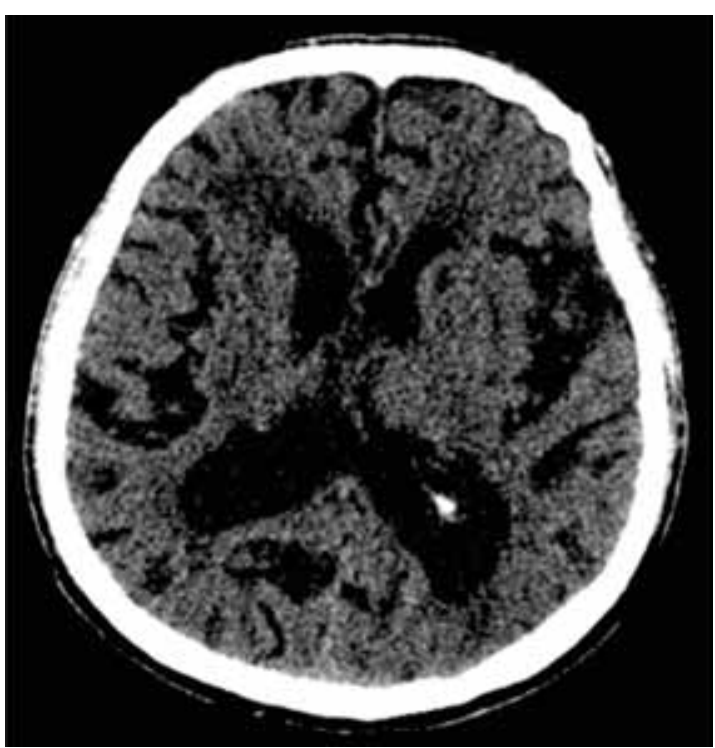

FIGURE 16. Mixed brain atrophy

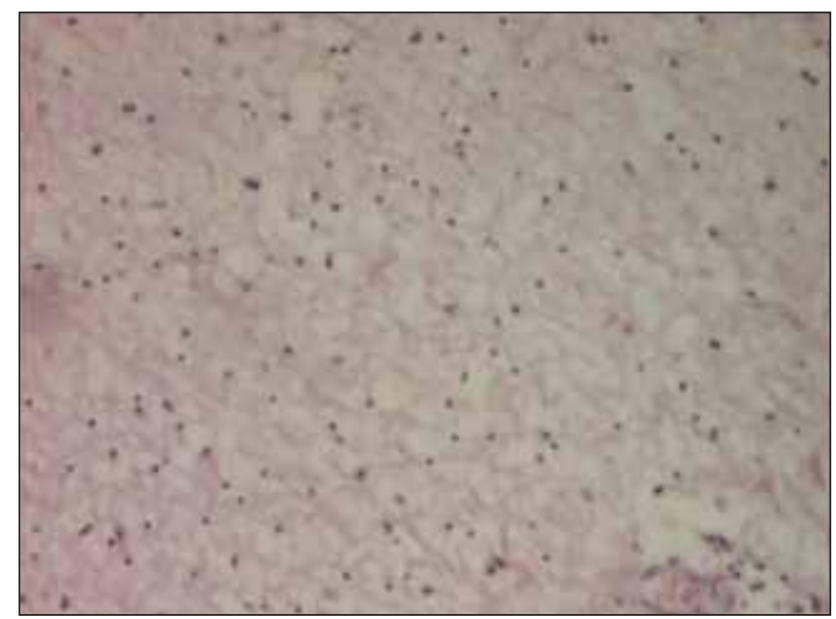

FIGURE 17. Cellular edema of the white matter (H.E. X 40)

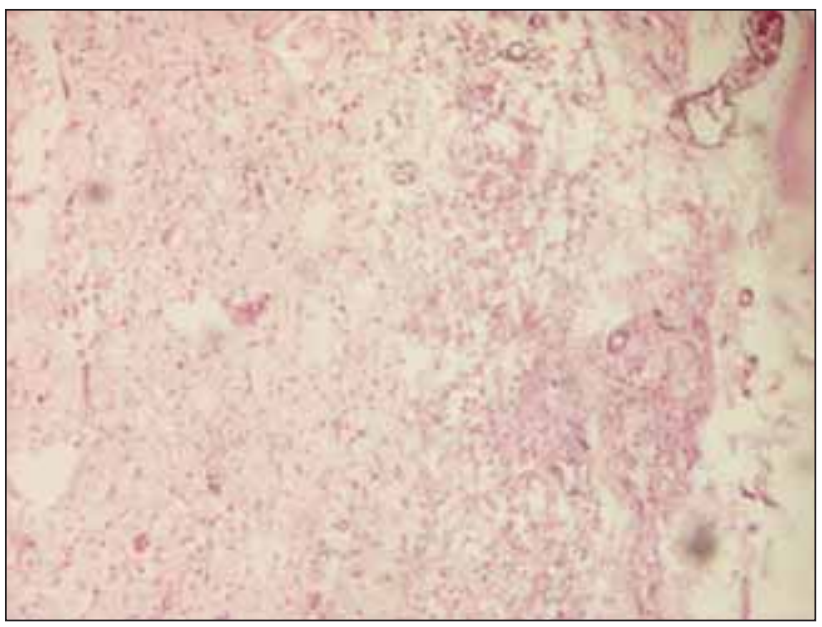

FIGURE 18. Marked vascular hyperemia of the meninges with subarachnoid space edema and mild acidophilia of the white matter (H.E. X 40)

\section{CONCLUSIONS}

There was no complete concordation between CT scan images and histological examination, as we cannot compare life and death (philosophically speaking). A possible explanation for this is that the CT scan was made in the first 24 hours, and the lesion was subsequently established.

Some of the limitations of the brain CT scan were compensated by MRI scan. In the study there are no MRI results to compare the results.

In the sudden death cases that had no acute lesions on the brain $\mathrm{CT}$ on admission, histological examination revealed the diagnosis. This is because sudden death is usually caused by heart diseases.

Histological examination in all the cases included in our study showed atherosclerotic modifications of cerebral and peripheral vessels (the circle of Willis arteries, coronary arteries, aorta and renal arteries). This is foreseeable, as the patients in the study are above 50 years of age.

The clinical, imagistic and histological correlations in the study of arteriosclerosis are very important due to the fact that cardiovascular disease and stroke are the main causes of death in developped countries.

\section{REFERENCES}

1. Ropper A.H. , Samuels M.A. - Adams and Victor s Principles of Neurology, ninth edition 2009

2. Harrison - Principiile Medicinei Interne $14^{\text {th }}$ edition 2003 pg. 1484-1492

3. Yousem D.M., Grossman R.I. - Neuroradiology: the Requisites, 3th Edition 2010, pg. 104-170

4. Taveras J.M. - Neuroradiology, 3th Edition, 1996, pg. 401-571

5. Arseni C. - Tratat de neurologie, IV partea I, Ed. Medicală, Bucureşti 1982, pg. 423- 428; 729-738

6. Popa C. - Problema accidentelor vasculare cerebrale grave, Revista Română de Stroke (AVC) vol.XIV Nr.1 2011, pg. 3-15

7. Kumar et. al - Robbins \& Cotran Pathologic Basis of Disease $7^{\text {th }}$ Edition 2005 pg.119-120; 137-139; 1361-1369

8. Arboix A., Grive E. - Intracerebral Hemorrhage: Influence of Topography of Bleeding on Clinical Spectrum and Early Outcome, Peter Bright: Neuroimaging - Methods; 2012 pg. 271-292

9. Duldner J.E. - Volume of intracerebral hemorrhage. A powerful and easy-to-use predictor of 30-day mortality. Stroke 1993; 24: 987-993

10. Sessa M. - Intracerebral hemorrhage and hypertension. Neurol Sci. 2008 Sep; 29 Suppl 2: S258-9. Review.

11. Popa C. - Boala aterosclerotică carotidiană. Elemente de histopatologie. Revista Română de Stroke (AVC) vol.X Nr.1 2007, pg. 11-26

12. Arsene D. - Neuropatologie; Ed. Didactică şi Pedagogică Bucureşti, 2002 pg. 37-83

13. Hancu A., Răşanu I. - White Matter Changes in Cerebrovascular Desease: Leukoaraiosis; V. Chaudhary: Advances in Brain Imaging; 2012, pg. 235-254

14. Lou Min, Al-Hazzani A. - Relationship Between White Matter Hyperintensities and Hematoma Volume and Growth in Patients with Intracerebral hemorrhage; Stroke 2010; 41: 34-40

15. Roger N. - Rosenberg: Atlas of Clinical Neurology, Second Edition, 2003, pg. 118-136; 175-176 